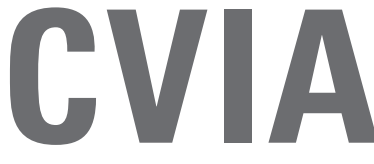

ERRATUM

pISSN 2508-707X / elSSN 2508-7088 https://doi.org/10.22468/cvia.2019.00094 CVIA 2019;3(3):99-99

\title{
Polycythemia Vera Presenting as Left Ventricular Pseudoaneurysm: The Role of Multimodality Imaging
}

Mohamad Jihad Mansour ${ }^{1}$, Ahmad Haddoul', Antranik Ohanian', Ahmad El Haj Sleiman², Raed Osman ${ }^{3}$, Atika Adhami ${ }^{3}$

'Division of Cardiology, Lebanese University, Faculty of Medical Sciences, Hadath, Lebanon ${ }^{2}$ Divisions of Cardiovascular and Thoracic Surgery,

${ }^{3}$ Cardiology, Rafik Hariri University Hospital, Beirut, Lebanon

CVIA 2019;3(2):56-59 / https://doi.org/10.22468/cvia.2019.00010

The original publication contains a typo: "onlone." The correct word is "online." 